\title{
Effect of Selective-task vs Set-task Program on Balance and Weight Bearing of Stroke Patient
}

\author{
Myung Hwan Ahn, MS, PT ${ }^{1)}$, Chang Sik Ahn, PhD, PT²), Myung Chul Kim, PhD, PT2) \\ 1) Rusk Rehabilitation Medical Center \\ ${ }^{2)}$ Department of Physical Therapy, Faculty of Health Science, Eulji University: \\ 212 Yangji-dong Sujeong-gu Seongnam-si Gyeonggi-do, 461-815 South Korea. \\ TEL:+82 31-740-7232,E-mail: acsik@eulji.ac.kr
}

\begin{abstract}
Purpose] This study investigated the change in functional balance and weight bearing of stroke patients in a set-task balance training program and a selective-task balance training program. [Method] the participants were randomly allocated to either the set-task program or the selective-task program. We evaluated balance capacity using the Berg Balance Scale (BBS). Postural weight bearing was measured using a Tetrax Intractive Balance System, and the lower extremity function was evaluated using the Fugel-Meyer Assessment of Sensorimotor Impairment (FMLE). Both programs were performed three days a week for $30 \mathrm{~min}, 8$ for weeks. The participants were assessed again at the end of the 8 weeks of training, and again after 3 months. [Results] The selective-task group showed significant improvments in BBS and plantar foot pressure after training. FMLS showed no significant difference in the set-task group after training; however, the selective-task group showed a significant improvement. At the 3-month follow-up BBS and Plantar foot pressure had maintained the significant improvement in both groups, but FMLS only showed a significant improvement in the selective-task group. [Conclusion] Based on this study, a selective-task program for stroke patients in which the patients choose tasks is more effective at improving balance, symmetric weight bearing by the lower extremities, and lower extremity function than a set-task program.
\end{abstract}

Key words: Stroke patient, Selective-task, Set-task

(This article was submitted Mar. 4, 2011, and was accepted Apr. 21, 2011)

\section{INTRODUCTION}

A stroke is a sudden loss of neurological function caused by an interruption of blood flow to the brain, and it is a common cause of disability in adults ${ }^{1)}$. Most stroke patients have a variety of disabilities such as motor, sensory, cognitive and emotive disabilities, and balance deficits with postural control disabilities caused by motor-sensory impairment result in serious problems in ADL for stroke patients ${ }^{2}$. Balance recovery after stroke is an important factor for daily living, so appropriate interventions with early assessment and treatment are needed to improve balance for functional recovery and successful rehabilitation. However, determining a specific cause is difficult because balance deficits after stroke are affected by various complex factors ${ }^{3)}$. It is therefore important to determine the main causal factor and carry out subsequent balance training ${ }^{4)}$. Although balance is needed to carry out a variety of simultaneous tasks in daily life, balance training is a relatively simple task.

Simple training uses a separated functional task used to sustain balance while standing, walking, transfer, etc. Putting a patient in various situations reinforces the learning of different balance tasks in various functional tasks.

It has been reported that training in various tasks is more effective for improving the walking speed, balance, endurance, function, etc of stroke patients ${ }^{5-7)}$. In the dualtask method, a patient is asked to carry out various tasks involving postural control. Recently, a task-oriented training method which aims to change CNS plasticity was conducted in which patients performed a functional task rather than repeating established training ${ }^{8}$. The circuit task-related approach is a clinical intervention for the re-education of stroke patients that is based on motor control and motor learning theory, and is also an effective way of keeping and improving function after discharge ${ }^{9,10)}$. Such methods allow a stroke patient to improve balance by improving the patient's internal or external motor control. Vereijiken et al. ${ }^{11)}$ indicated that external concentration focusing on the result of a movement facilitates automatic processing of motor control, while internal concentration focusing on the movement itself facilitates a conscious process. It is necessary to trigger the patient's motivation in the rehabilitation program and that motivation is an important factor for comprehensive rehabilitation.

Accordingly, this study investigated the changes in functional balance and weight bearing of stroke patients performing a set-task balance training program with three tasks and a selective-task program that allowed the patient to choose among the three tasks. Also, the maintenance 
Table 1. General characteristics of participants $n=30$

\begin{tabular}{lcccc}
\hline & & Set-task program $(\mathrm{n} 1=15)$ & Selective-program $(\mathrm{n} 2=15)$ & $\mathrm{P}$ \\
\hline Sex & Men & 7 & 9 \\
Age (year) & Women & 8 & 6 \\
Height $(\mathrm{cm})$ & & $67.5 \pm 1.8$ & $67.9 \pm 1.3$ \\
Weight $(\mathrm{kg})$ & & $52.1 \pm 8.9$ & $158.1 \pm 1.7$ \\
Onset (month) & & $10.3 \pm 9.6$ & $49.3 \pm 6.2$ \\
Hemiplegic side & Left & 8 & $11.3 \pm 11.0$ \\
& Right & 7 & 9 \\
\hline
\end{tabular}

$* \mathrm{p}<0.05$

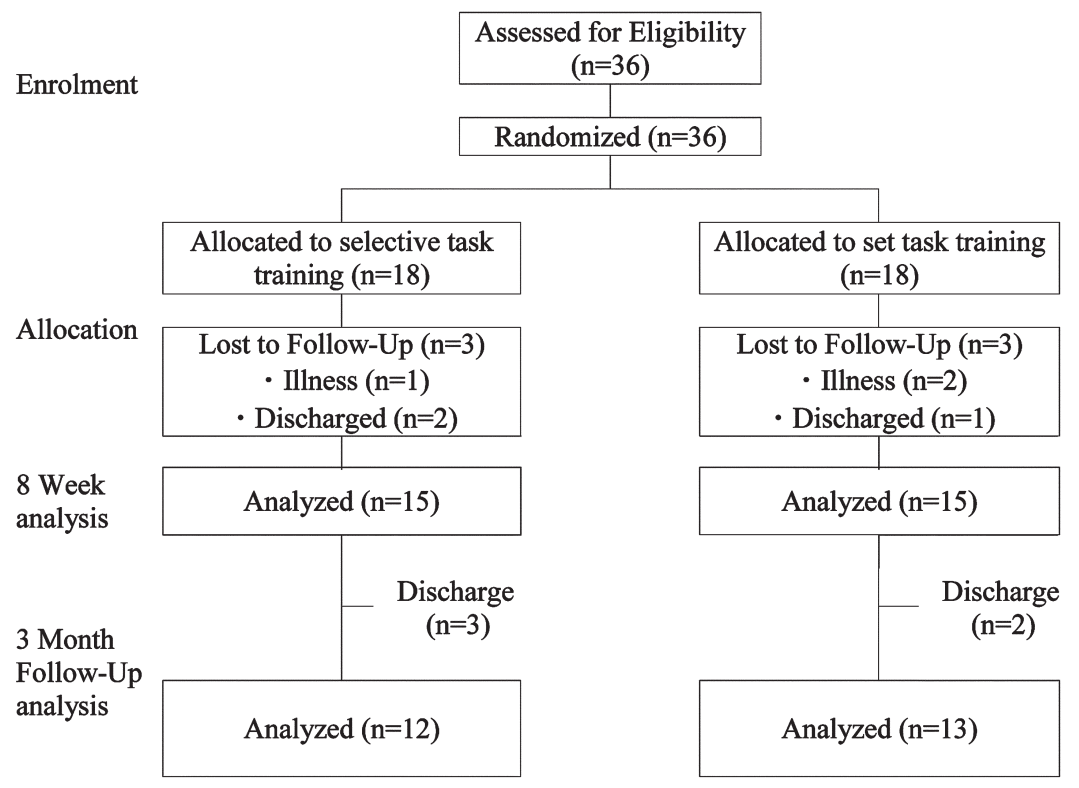

Fig. 1. Diagram of experimantal procedure in this styudy

effect with exercise was verified by reassessment after 3 months.

\section{SUBJECTS AND METHODS}

The participants were inpatients diagnosed with hemiplegia from stroke of a rehabilitation hospital located in Yongin City. Although the study began with 36 participants, three in the set-task program and three in the selective-task program could not attend regularly or were discharged. Therefore, only 30 participants were included in the analysis of this experiment. The study was conducted from March to May 2010. To participate, subjects had to meet the following inclusion criteria: clinical diagnosis of a first stroke; within 24 months post stroke; able to attend the program performed three days a week for $30 \mathrm{~min}$, for 8 weeks ; able to walk 10 meters independently with or without an assistive device; sufficient hearing and vision; score at least 24 on MMSE-K(Mini-mental State Examination - Korea version ; MMSE-K); no orthopedic problems with either knee and no complications that interfered with balance; and agreed to participation in the experiment. Subjected were excluded if they had any medical condition that would prevent participation in a training program.

The general characteristics of the participants, 7 males and 8 females, were a mean age of at $67.53 \pm 1.80$ a mean height of , $156.13 \pm 8.87 \mathrm{~cm}$, and a mean weight of $52.93 \pm$ $7.01 \mathrm{~kg}$ in the simple-task program group; and there were 9 males and 6 female aged $67.93 \pm 1.27$, with a mean height of and mean weight of $158.13 \pm 1.65 \mathrm{~cm}$, and mean weight of $49.26 \pm 6.19 \mathrm{~kg}$ in the selective-task group (Table 1). Since the study was carried out at two separate locations, the randomization was done in blocks.

The participants were randomly allocated to either the set-task program or the selective-task program. Both the set task and the selective task groups received standard physiotherapy such as gait training and neurological developmental therapy five days a week, as well as the task program three days a week for $30 \mathrm{~min}$. The therapy period was 8 weeks. The participants were assessed after the 8 weeks of training, and again after 3 months (Fig. 1). Taskrelated training for this study followed the studies of Dean et al. ${ }^{12)}$ and Salbach et al. ${ }^{13)}$. A manual control task in the 
Table 2. Comparison of all measures between groups during training

\begin{tabular}{|c|c|c|c|c|}
\hline Variable & Measurement time & $\begin{array}{l}\text { Set-task program } \\
(\mathrm{n}=15)\end{array}$ & $\begin{array}{l}\text { Selective-task program } \\
\qquad(\mathrm{n}=15)\end{array}$ & \\
\hline \multirow{5}{*}{ BBS } & 0 & $30.3 \pm 2.9$ & $30.5 \pm 2.3$ & \\
\hline & 8 week & $31.5 \pm 1.9$ & $32.9 \pm 1.7$ & $*$ \\
\hline & $3 \mathrm{mon} F / \mathrm{U}$ & $29.5 \pm 2.0$ & $31.3 \pm 1.9$ & $*$ \\
\hline & F & 21.1 & 17.9 & - \\
\hline & & $*$ & $*$ & - \\
\hline \multirow{5}{*}{ Plantar Foot Pressure } & 0 & $21.7 \pm 3.3$ & $19.4 \pm 8.8$ & \\
\hline & 8 week & $15.2 \pm 7.3$ & $10.8 \pm 3.7$ & $*$ \\
\hline & 3 mon $\mathrm{F} / \mathrm{U}$ & $15.5 \pm 6.4$ & $10.2 \pm 6.1$ & $*$ \\
\hline & F & 18.5 & 13.5 & - \\
\hline & & $*$ & $*$ & - \\
\hline \multirow{5}{*}{ FMLS } & 0 & $14.7 \pm 2.9$ & $14.1 \pm 3.0$ & \\
\hline & 8 week & $15.7 \pm 2.8$ & $20.1 \pm 3.8$ & $*$ \\
\hline & $3 \mathrm{mon} F / \mathrm{U}$ & $14.3 \pm 2.1$ & $17.5 \pm 2.8$ & $*$ \\
\hline & F & 2.2 & 9.6 & - \\
\hline & & & $*$ & - \\
\hline
\end{tabular}

BBS, Berg Balance Scale; FMLS, Fugel-Meyer Assessment of Sensorimotor Impairment. *p<0.05.

standing posture was performed for standing balance, weight shifting, reinforcing coordination and improving balance. The therapist minimized feedback to the patient to facilitate motor learning.

The detailed training program was as follows: 1) To put a bean into a wooden box and stand with feet shoulderwidth apart or with knees together, and to bend down with one hand or both hands to the face as if washing up; 2) To do the ironing forward to backward and right to left, standing with feet shoulder-width apart; 3) To pull a heel up and down while cleaning a mirror standing with feet shoulder-width apart; 4) To pull the heel up and down with a stick as if painting; 5) To shoot a ball forward into a target and sideways within reach of a safety bar for weight shifting; 6) To rub a towel on the floor within reach of a safety bar for weight shifting; 7) To reach the knee or foot to various heights for weight shifting; 8) To throw a ring onto a stick from various directions for coordination of upper and lower extremities; 9) To toss and catch a shuttlecock while playing badminton for coordination of upper and lower extremities; ${ }^{10}$ To throw darts at a target while standing.

All tasks were carried out for $10 \mathrm{~min}$. Each patient in the set-task program carried out the 2 nd standing task, the 5 th weight shifting task, and the 9th coordination task of upper and lower extremities. The set-task program modified and utilized activities which are commonly encountered in the home and in the community. In contrast, the patients in the selective-task program were allowed to choose their training programs: one from tasks $1 \sim 4$ for standing, one from tasks $5 \sim 7$ for weight shifting, and one from tasks $8 \sim 10$ for coordination of upper and lower extremit.

For assessment, we used the Berg Balance Scale, Plantar Foot Pressure Distribution and the Lower-extremity subscale of the Fugel-Meyer Assessment of Sensorimotor Impairment (FMLE).

The Berg Balance Scale (BBS) was used to measure balance. BBS is a tool that makes it possible to quantify balance capacity in tasks such as standing with eyes closed, standing with knees together, and while lifting an object ${ }^{14)}$. It scores items from 0 to 4 giving a total ranging from 0 to 56 . BBS has high interexaminer reliability $\left(\mathrm{r}^{2}=0.98\right)$ and test-retest reliability $\left(\mathrm{r}^{2}=0.99\right)^{15)}$.

Postural weight bearing was measured using a Tetrax Intractive Balance System (Tetrax, Ramat Gan, and Sunlight Medical, Tel-Aviv, Israel). This method of posturography is based on the assessment of the vertical pressure fluctuations on four independent force plates placed beneath the heels and toes of the subject while standing in an upright position. The software of the system elaborates four basic parameters obtained in the standing positions.

Belgen, Beninato, Sullivan and Narielwalla ${ }^{16)}$ used FMLE to measure body function influence on balance in stroke patients. FMLE is a tool for measuring the lower extremity function of a subject. Each item is scored from 0 to 2 giving a total ranging from 0 to 34 . It is possible to measure the reflex, motor, synergy, and coordination of the lower extremity with FMLE which has high interexaminer reliability $\left(r^{2}=8.89\right)$ and test-retest reliability $\left.\left(r^{2}=0.96\right)^{15}\right)$.

SPSS version 17.0 was used to analyze the data and the independent t-test was used to test the homogeneity of the two groups. Repeated analysis of variance was used to analyze the changes with time in the exercise groups. LSD was used for post hoc analysis. The level of statistical significance was shosen as 0.05 .

\section{RESULTS}

There were no significant differences in the homogeneity of the general characteristics of the participants, sex, age, height, weight, onset and paralyzed part. The comparison of dynamic balance assessed by the Berg Balance Scale (BBS) in pre- and post-training is shown in Table 2. 
The pre-training dynamic balance was $30.33 \pm 2.91$ in the set-task program and $30.46 \pm 3.32$ in the selective-task group. The post-training dynamic balance was $31.46 \pm 1.92$ with an average increase of 1.13 in the set-task program and was $32.86 \pm 1.72$ with an average increase of 2.40 in the selective-task program. At the 3-month follow-up, both the selective-task program group and the set-task program group showed statistically significant decreases of BBS from its post-traning value $(\mathrm{p}<0.05)$.

The comparison of the change in symmetric plantar foot pressure is shown in Table 2. The pre-training plantar foot pressure was $21.67 \pm 3.30$ in the set-task group and $19.36 \pm$ 8.97 in the selective-task group. Post-training, it was 15.20 \pm 7.32 in the set-task program group, an average decrease of 6.47 from asymmetric to symmetric, while in the selective-task group, it was $10.86 \pm 3.68$, an average decrease of 8.56. At the 3-month follow-up, showed significant decreases from the pre-training level both the selective-task program group and the set-task program group $(\mathrm{p}<0.05)$.

The comparison of lower extremity function with preand post-training and after 3 months is shown in Table 2 . The lower extremity function value at pre-training was $14.73 \pm 2.86$ in the set-task group and $14.06 \pm 3.01$ in the selective-task group. Post-training, it was $15.66 \pm 2.84$, an average increase of 0.93 in the set-task group, and $20.13 \pm$ 3.83 , an average increase of 6.07 in the selective-task group. At the 3-month follow-up, there were significant differences from the pre-/post-training values in both the selective-task group and the set-task group $(\mathrm{p}<0.05)$.

Most participants in the selective task program selected item 4 from among the standing tasks, item 7 from among the weight shifting tasks and item 10 from among the coordination tasks for the upper and lower extremities.

\section{DISCUSSION}

Motor control is defined as the ability to control movement directly and regularly via the CNS system. A complex movement is defined as an interaction between personal situation, environment and appropriate task performance ${ }^{10)}$. Generally, hemiplegic patients with balance and weight-shifting problems have impairments of upright position and gait because of asymmetric posture, abnormal body balance and difficulty in shifting weight ${ }^{17}$.

In this comparative study of a selective-task program and a set-task program, there were significant improvements in dynamic balance, symmetric plantar foot pressure distribution and change of lower extremity function in the selective-task group $(\mathrm{p}<0.05)$. Consequently, the selectivetask program was effective al improving the balance of hemiplegic patients.

In balance control during task performance, internal concentration movement is interrupted by automatic movement control, resulting in conscious balance control. Concentration on task performance facilitates effective information processing and initiates automatic and reactive movement control enhancing motor learning and performance ability ${ }^{18}$. Wulf et al. ${ }^{19)}$ mentioned that greater postural control is required for upper extremity tasks.

In the selective-task group, the Berg Balance Scale for dynamic balance showed a statistically significant improvement. In the set-task group, BBS was $30.33 \pm 2.91$ before exercise and increased to $31.46 \pm 1.92$ after exercise, while it was $30.46 \pm 2.32$ before exercise and increased to $32.86 \pm 1.72$ after exercise in the selective-task group. The plantar foot pressure distribution test for horizontal weight bearing distribution of the lower extremity also showed a statistically significant improvement in the selective-task group. It was $21.67 \pm 3.30$ before exercise and decreased to $15.20 \pm 7.32$ after exercise in the set-task control group, and it was $19.36 \pm 8.79$ before exercise, decreasing to $10.80 \pm$ 3.68 after exercise in the selective-task experimental group. The FMLS assessment of lower extremity function showed no significant difference in the set-task control group being $14.73 \pm 2.86$ before exercise and $15.66 \pm 2.84$ after exercise. However, there was a significant improvement the selective-task group form $14.06 \pm 3.01$ before exercise to $20.13 \pm 3.13$ after exercise. This result was in agreement with the study by Stoffregen et al. ${ }^{20}$ which reported decreased postural sway when patients performed balance control using the lower extremity with a building block on the upper extremity, and with the study by Riley et al. ${ }^{21)}$ which reported decreased body sway patients performed balance control with a secondary task compared to only maintaining balance of the lower extremity. Hoo Sung and Park $^{22)}$ mentioned that the effort to solve a varied task within a given environment requires autonomic body control with proper stability while performing the task because the process involves simultaneous balance training in a dual-task.

In all exercise methods for stroke patients, task-centered treatment is more effective than strengthening exercises ${ }^{23,24)}$ or the general approach described by Eun Jung and Choi ${ }^{25)}$ which used a psychologically centered exercise program involving volitional participation and stimulation of motivation. This study was conducted to elucidate the advantages and effects of a selective-task program by comparison with a set-task program. In the selective-task program, participants volitionally chose exercises from a 10 -item exercise program. Therefore, the development of an intervention plan to improve upright posture and dynamic balance of hemiplegic patients that includes a selective program that allows the patients to concentrate, and stay immersed and interested in the program is necessary. To improve function of stroke patients, there must be a relationship between the functional activity performed and a self-performed activity in real life. The results of this study show a selective-task program for stroke patients in which the patient chose tasks was more effective at improving balance, symmetric weight bearing lower extremities, and lower extremity function then a settask program.

More research is needed to develop a more systemic and effective exercise program based on expert knowledge that can be available to more patients and is easily carried out in real life. 


\section{REFERENCES}

1) Adamson J, Beswick A, Ebrahim S: Is stroke the most common cause of disability? J Stroke Cerebrovasc Dis, 2004, 13: 171-177.

2) De Haart, M Geurts, AC Huidekoper, et al.: Recovery of standing balance in post acute stroke patients: a rehabilitation cohort study. Arch Phys Med Rehabil, 2004, 85: 886-895.

3) Horak FB: Clinical assessment of balance disorders. Gait Posture, 1997, 6: 76-84.

4) Tyson SF, Hanley M, Chillala J, et al.: Balance disability after stroke. Phys Ther, 2006, 86: 30-38.

5) Dean CM, Shephard RB: Task-related training improves performance of seated reaching tasks after stroke. Stroke, 1997, 28: 722-728.

6) Silsupadol P, Shumway-Cook A, Woollacott MH, et al.: Training of balance under single-and dual-task conditions in older adults with balance impairment. Phys Ther, 2006, 86: 269-281.

7) Wu CY, Wong MK, Lin $\mathrm{KC}$, et al.: Effects of task goal and personal preference on seater reaching kinematics after stroke. Stroke, 2001, 32: 7076.

8) Johansson BB: Brain plasticity and stroke rehabilitation. The Willis lecture. Stroke, 2000, 31: 223-230.

9) Dean CM, Richards CL, Malouin F: Task-related circuit training improves performance of locomotor tasks in chronic stroke: A randomized, controlled pilot trial. Arch Phys Med Rehabil, 2000, 81: 409-417.

10) Shumway-Cook A, Wollacott MH: Motor control translating research into clinical practice (3rd ed). Lippincott Williams \& Wilkins, 2007.

11) Vereijken B, van Emmerik RE, Whiting HT, et al.: Freezing degrees of freedom in skill acquisition. J Mot Behav, 1992, 24: 133-142.

12) Dean $\mathrm{CM}$, Richards $\mathrm{Cl}$, Malouin F.: Task-related circuit training improves performance of locomotor tasks in chronic stroke: Arandomized, controlled pilot trial. Arch Phys Med Rehabil, 2000, 81: 409-417.

13) Salbach NM, Mayo NM, Wood Dauphinee S, et al.: A task orientated intervention enhances walking distance and speed in the first year post stroke: a randomized controlled trial. Clin Rehabil, 2004, 18: 509-519.
14) Silsupadol P, Shumway-Cook A, Lugade V, et al.: Effect of single-task versus dual-task training on balance performance in older adults: A doubleblind, randomized controlled trial. Arch Phys Med Rehabil, 2009, 90: 381387.

15) Beninato M, Portney LG, Sullivan PE: Using the international classification of functioning, disability and health as a framework to examine the association between falls and clinical assessment tools in people with stroke. Phys Ther, 2009, 89: 816-825.

16) Belgen $B$, Beninato $M$, Sullivan PE, et al.: The association of balance capacity and falls self-efficacy with history of falling in communitydwelling people with chronic stroke. Arch Phys Med Rehabil, 2006, 87: 554-561.

17) Bobath B: Adult Hemiplegia Evaluation and treatment (3rd ed). London: Butterworth-Heinemann Medical Books. 1990.

18) Roh JS, Lee CW, Cho SH, et al.: The effect of attentional focus on the performance of dual task. Phys Ther Korea, 2008, 15: 11-19.

19) Wulf G, Mercer J, McNevin N, et al.: Reciprocal influences of attentional focus on postural and suprapostural task performance, 2004, 36: 189-199.

20) Stoffregen TA, Pggulayan RJ, Bardy BG, et al.: Modulating postural control to facilitate visual performance. Human Mocement Science, 2000, 19: 203 220.

21) Riley JM, David B, Kaber: Adaptive automation of a dynamic control task based on secondary task workload measurement. International Journal of Cognitive Erognomics, 1999, 3: 169-187.

22) Park HS: The effect of balance training under single task and dual task conditions in older adults. Korea sport research, 2007, 18: 109-120.

23) Leroux A, Pinet H, Nadeau S: Task-oriented intervention in chronic stroke: Changes in clinical and laboratory measures of balance and mobility. Am J Phys Med Rehabil, 2006, 85: 820-830.

24) Lin $\mathrm{K}, \mathrm{Wu} \mathrm{C}$, Chen $\mathrm{C}$, et al.: Effects of object use on reaching and postural balance: A comparison of patients with unilateral stroke and healthy controls. Am J Phys Med Rehabil, 2007, 86: 791-799.

25) Choi EJ: Effects of rehabilitation program based on self-efficacy on poststroke patients. Seoul University. Dissertation of Doctorte Degree, 2002 . 